\title{
Diabetes como manifestación de infección de Hemocromatosis Hereditaria
}

\author{
Pablo Franquelo Morales ${ }^{a}$, Félix González Martínez ${ }^{\mathrm{a}}$, \\ Mubarak Alramadan Alramadan ${ }^{b}$
}

a Servicio de Urgencias del Hospital Virgen de la Luz. Cuenca.

\section{b Servicio de Endocrinología del Hospital Virgen de la Luz. Cuenca.}

\section{Correspondencia:}

Pablo Franquelo Morales.

Servicio de Urgencias

del Hospital Virgen de la Luz. $\mathrm{C} /$ Hermandad de

Donantes s/n, 16002-Cuenca.

E-mail: pfranquelo@yahoo.es

Recibido el 8 de enero de 2008.

Aceptado para su publicación el 25 de enero de 2008.

\section{RESUMEN}

La Hemocromatosis hereditaria $(\mathrm{HH})$ es un desorden genético común del metabolismo del hierro de carácter recesivo. Los pacientes con $\mathrm{HH}$ presentan una mayor absorción intestinal de hierro y éste puede acumularse en grandes cantidades provocando disfunción orgánica. La frecuencia de diabetes está aumentada en la $\mathrm{HH}$. El diagnóstico clínico se establece frecuentemente en base a parámetros de sobrecarga de hierro (índice de saturación de transferrina y ferritina sérica), sin embargo, con frecuencia es necesario realizar una biopsia hepática y determinar la cantidad de hierro en hígado para confirmar la presencia de depósitos de hierro. El diagnóstico de $\mathrm{HH}$ antes de que se produzcan depósitos de hierro es difícil y es necesario aumentar el grado de sospecha por parte del médico. Con un diagnóstico precoz y un tratamiento adecuado, la supervivencia de los pacientes es normal.

Palabras clave. Hemocromatosis. Diabetes mellitus.

\section{ABSTRACT}

Diabetes as an initial manifestation of Hereditary Hemochromatosis.

Hereditary hemochromatosis $(\mathrm{HH})$ is an inherited disorder of iron metabolism. It is one of the most common autosomal recessive conditions. In patients with $\mathrm{HH}$ the amount of iron absorbed by the intestines is increased, which may lead to excess iron being deposited in different organs of the body, causing organ dysfunction. The frequency of diabetes is increased in $\mathrm{HH}$. A clinical diagnosis of $\mathrm{HH}$ can often be established on the basis of blood tests (transferrin saturation index and serum ferritin level), however, a liver biopsy to determine the amount of iron in the liver is often required to confirm tissue iron overload. The diagnosis of hereditary hemochromatosis before iron overload has developed is difficult. However, doctors need to have a higher awareness of $\mathrm{HH}$ because with early diagnosis and appropriate treatment, survival of patients is normal.

Key Words. Hemochromatosis. Diabetes mellitus.

\section{INTRODUCCIÓN}

Disponer de unos criterios estrictos provoca que la prevalencia estimada de hemocromatosis hereditaria $(\mathrm{HH})$ en la población general sea muy variable ${ }^{1}$, no obstante se estima que ocurre en aproximadamente 5 de cada 1000 personas de raza blanca, considerándose como la enfermedad genética más frecuente en la población humana de origen caucasiano ${ }^{2}$. Es un trastorno frecuente en el que el incremento de los depósitos de hierro origina una lesión con alteraciones funcionales en el hígado, páncreas, articulaciones, corazón e hipófisis ${ }^{3}$. Su asociación con la diabetes es un hecho demostrado ${ }^{4}$, manifestándose en un 53\%-80\% de los casos como una complicación².

\section{OBSERVACIONES CLÍNICAS}

Varón de 43 años que acudió a la consulta de endocrinología remitido por su médico de atención primaria tras objetivar en análisis rutinario alteraciones del perfil lipídico y una glucemia basal $>200 \mathrm{mg} / \mathrm{dL}$ sin síntomas cardinales. No presentaba antecedentes patológicos de interés. La exploración física era normal, a excepción de un índice de masa corporal de 30 y un aumento llamativo de la pigmentación cutánea. En la analítica se hallaron los siguientes valores: glucosa 123 mg/dL, hemoglobina glicada (HbA1c) 7,4\%, transferrina $251 \mathrm{mg} / \mathrm{dL}$, índice de saturación de la transferrina 47\%, y ferritina en suero $555 \mu \mathrm{g} / \mathrm{L}$. Fue diagnosticado de DM tipo 2, obesidad grado I y aumento de la sideremia. Tras realizarse estudio genético por PCR, se diagnosticó he- 
mocromatosis hereditaria con genotipo $\mathrm{C} 282 \mathrm{Y} / \mathrm{H} 63 \mathrm{D}$ como probable origen de la diabetes. El paciente está en tratamiento desde entonces con flebotomías periódicas.

\section{DISCUSIÓN}

El depósito de hierro en hígado y páncreas provoca un aumento de la resistencia a la insulina y disminución de su secreción, constituyendo un síndrome clínico con afectación multiorgánica ${ }^{4}$. Aunque los síntomas clásicos de hemocromatosis como DM, hepatomegalia y pigmentación cutánea son importantes, la demostración de un incremento del porcentaje de saturación de la transferrina y la ferritina sérica (tabla1), constituyen el método diagnóstico más sencillo y adecuado en la fase inicial. Cada vez más se de- tecta como hallazgo incidental en relación con otras patologías $^{3}$, en las que se aprecian analíticas con parámetros de sobrecarga férrica elevados. Su asociación con diabetes, en un $10-30 \%^{3}$, ha disminuido a expensas de una detección más precoz, con una prevalencia actual alrededor del $9 \%^{5}$. Sin embargo, no se dispone de acuerdo acerca de los límites analíticos para su diagnóstico ${ }^{6}$. Los hallazgos por sí solos obligan a iniciar tratamiento, previa realización de biopsia hepática. No obstante, desde la existencia de pruebas genéticas, se ha visto desplazada. Queremos mostrar al médico de atención primaria la importancia de una alta sospecha clínica ante síntomas inespecíficos y de no desestimar los resultados anormales de la pruebas de laboratorio, disminuyendo así la morbimortalidad $^{3}$.

\begin{tabular}{l|c|c}
\hline Sexo & $\%$ Saturación de transferrina & Ferritina sérica \\
\hline Hombres & $\geq 55 \%$ & $>300 \mu \mathrm{g} / \mathrm{L}$ \\
\hline Mujeres posmenopáusicas & $\geq 55 \%$ & $>200 \mu \mathrm{g} / \mathrm{L}$ \\
\hline Mujeres premenopáusicas & $\geq 45 \%$ & $>200 \mu \mathrm{g} / \mathrm{L}$ \\
\hline
\end{tabular}

Tabla 1. Valores analíticos del hierro en la hemocromatosis.

\section{BIBLIOGRAFÍA}

1. Phatak PD, Sham RL, Raubertas RF, Dunnigan K, O'Leary MT, Braggins C et al. Prevalence of hereditary hemochromatosis in 16031 primary care patients. Ann Intern Med. 1998; 129(11):954-61.

2. De Portugal Alvarez J. Hemochromatosis: from phenotype to genotype. An Med Interna. 2002; 19(4):163-5.

3. Del Castillo Rueda A, López-Herce Cid JA, De Portugal Álvarez J. Hemocromatosis hereditaria. Diagnóstico clínico: manifestaciones precoces, procesos relacionados y formas atípicas. An Med interna (Madrid) 2002; 19:251-256.

4. Swaminathan S, Fonseca VA, Alam MG, Shah SV. The role of iron in diabetes and its complications. Diabetes Care. 2007; 30(7):1926-33.

5. Powell LW, Subramaniam VN, Yapp TR. Haemochromatosis in the new millennium. J Hepatol. 2000; 32(1 Suppl):48-62.

6. Qaseem A, Aronson M, Fitterman N, Snow V, Weiss KB, Owens DK. Screening for hereditary hemochromatosis: a clinical practice guideline from the American College of Physicians. Ann Intern Med. 2005; 143(7):517-21. 